Delft University of Technology

\title{
The Soft Inverted Pendulum with Affine Curvature
}

\author{
Santina, Cosimo Della
}

DOI

10.1109/CDC42340.2020.9303976

Publication date

2020

Document Version

Final published version

Published in

Proceedings of the 59th IEEE Conference on Decision and Control, CDC 2020

\section{Citation (APA)}

Santina, C. D. (2020). The Soft Inverted Pendulum with Affine Curvature. In Proceedings of the 59th IEEE Conference on Decision and Control, CDC 2020 (pp. 4135-4142). IEEE .

https://doi.org/10.1109/CDC42340.2020.9303976

\section{Important note}

To cite this publication, please use the final published version (if applicable).

Please check the document version above.

Other than for strictly personal use, it is not permitted to download, forward or distribute the text or part of it, without the consent of the author(s) and/or copyright holder(s), unless the work is under an open content license such as Creative Commons.

\section{Takedown policy}

Please contact us and provide details if you believe this document breaches copyrights.

We will remove access to the work immediately and investigate your claim. 
Green Open Access added to TU Delft Institutional Repository

'You share, we take care!' - Taverne project

https://www.openaccess.nl/en/you-share-we-take-care

Otherwise as indicated in the copyright section: the publisher is the copyright holder of this work and the author uses the Dutch legislation to make this work public. 


\title{
The Soft Inverted Pendulum with Affine Curvature
}

\author{
Cosimo Della Santina ${ }^{1,2,3}$
}

\begin{abstract}
We introduce here a novel under-actuated mechanical system motivated by recent advances in soft robotics. We derive its governing equations, discuss its properties, and consider a solution for the stabilization of its unstable equilibrium. The results we propose here are intended as a first step towards dealing with the much more challenging general problem of controlling full fledged soft robots subject to non negligible external forces and operating at high accelerations.
\end{abstract}

\section{INTRODUCTION}

Continuum soft robots [1] are mechanical systems made of continuously deformable soft materials, equipped with a finite set of actuators acting at discrete points along the structure. The last decade saw an exponential growth in the development of soft robotic platforms with increasing motion and actuation capabilities. Nonetheless, their ability of executing complex tasks is still very limited, due to the overwhelming complexity of the associated control problem. The reasons behind this impasse are of both technological and theoretical kind. We are interested here in the latter, for which the main source of complexity comes from the infinite dimensional nature of soft robots, and the consequent strong under-actuation.

Attacking the control problem directly from an infinite dimensional standpoint seems excessive at the current level of the state of the art. Indeed, control of partial differential equations is still largely focused on linear systems. Although inspiring, results concerning the nonlinear case are still of limited applicability [2], [3], not yet quite matching the complexity of a soft robot [4]. As a consequence, it has been for long thought that machine learning was the only sensible approach to soft robotic control [5], and model based strategies were relegated to quasi-static kinematic control [6]. Nevertheless, this scenario is rapidly evolving thanks to the development of accurate yet manageable finite dimensional models of soft robots [7], [8]. This in turn is driving the study of model-aware control strategies, and ultimately the establishment of soft robotic control as a proper sub-field of control theory.

First important results going in this direction deal with soft robots for which the dynamic effect of continuum parts is negligible [9]. The dynamic contributions of nonlocalized deformations are instead taken into account by [10], [11]. While working well in the practice, all these techniques rely on a fully actuated reduced model of the robot. This simplifying assumption clearly exposes the closed loop to control-spillover, and more in general to erroneous assessment of stability properties and performance.

\footnotetext{
${ }^{1}$ Department of Cognitive Robotics, Delft University of Technology, Delft, The Netherlands, ${ }^{2}$ Institute of Robotics and Mechatronics, German Aerospace Center (DLR), Oberpfaffenhofen, Germany, ${ }^{3}$ Department of Informatics, Technical University of Munich, Munich, Germany. Contact cosimodellasantina@gmail.com.
}

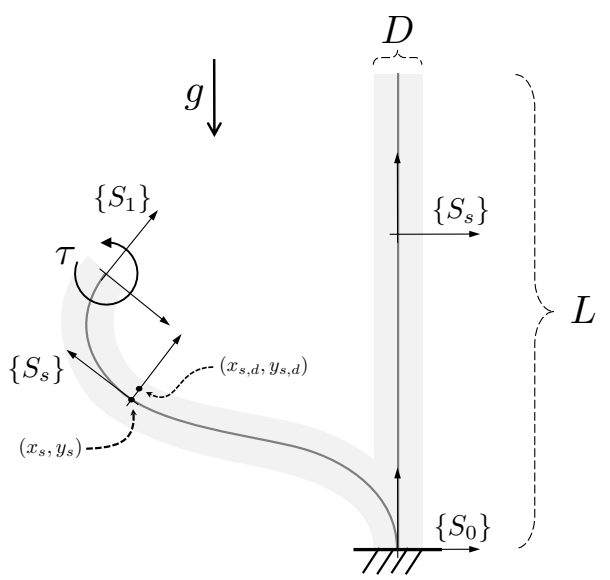

Fig. 1. The soft inverted pendulum with main quantities highlighted. The principal axis is shown as a dark gray line. The system is subject to gravity, and it is actuated trough a pure torque $\tau$ applied at its tip. Its curvature at any point is an affine function of the the coordinate identifying the position along the main axis of the robot. The weights of this function are the configuration variables of the system.

This issue has been faced by [12], albeit within a linear approximation. Here, stability is proven for a proportional feedback loop acting on an high dimensional FEM model through a reduced number of control inputs. The underactuated and fully nonlinear nature of the control problem is instead faced in [13], where the PD-poly is proposed, as an extension of the $\mathrm{PD}$ regulator taking into account higher order deformation modes.

The present paper goes deeper into this challenge by introducing a novel under-actuated template system, the soft inverted pendulum with affine curvature. Fig. 1 depicts the pendulum in two configurations. This model can be seen as the soft counterpart of the well-knownn acrobot [14]. Here, the two degrees of freedom are distributed along the soft structure rather than being concentrated in two joints. To achieve this goal, we contextually introduce an analytical model for soft robots extending our previous work [13]. In analogy with the acrobot, the control action is considered here applied the farthest from the base. This choice is motivated by the kind of actuation sources that we encounter in soft robotics [11]. The main properties of this system are discussed, and nonlinear feedback controllers are developed regulating the unstable straight configuration of the robot.

Our approach substantially mirrors the path that the control community undertook in the 90's and early 00's, when trying to understand how to deal with under-actuated rigid robots. Rather than attacking directly the general problem, a vast range of low dimensional template models were introduced and their behavior studied in detail [15]. Examples include the cart pole, the pendubot, and the above mentioned acrobot [14]. The impact in robotics of the fruits of these efforts cannot be overstated. On the one hand, they have provided theoretical insights about what and how 


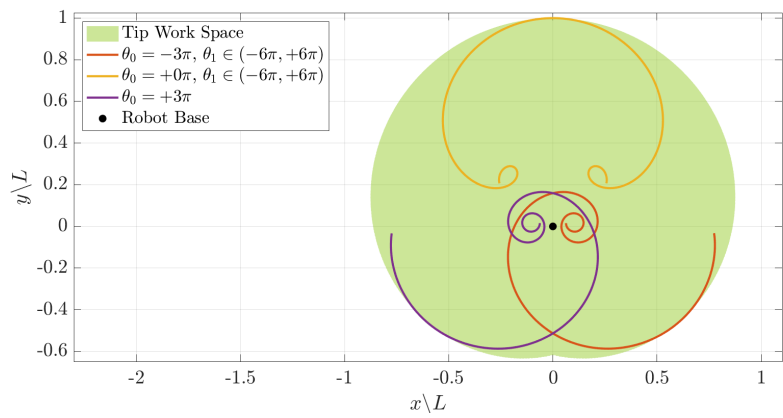

(a) Workspace

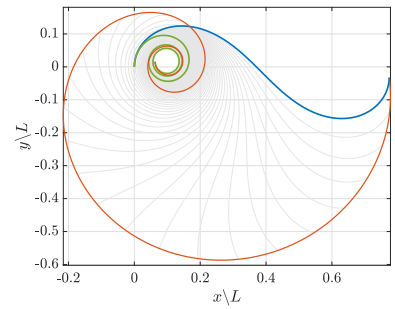

(b) $\theta_{0}=-3 \pi$

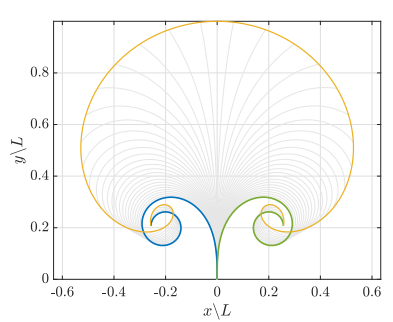

(c) $\theta_{0}=0$
Fig. 2. Analysis of the positions that can be attained by the pendulum's tip, according to (5) and for $\theta_{0}, \theta_{1} \in(-6 \pi, 6 \pi)$. Panel (a) shows the complete workspace, also presenting three curves that are traced for selected constant values of $\theta_{0}$, and $\theta_{1}$ varying in the whole considered range. Corresponding configurations of the pendulum are shown within panels (b) and (c). The loci of tip positions are color coded as in panel (a). The shape of the pendulum for $\theta_{1}=-6 \pi$ is shown in green, and for $\theta_{1}=+6 \pi$ in blue. Intermediate configurations are plotted in light gray.

can be achieved with underactuated mechanical systems. On the other hand, their direct implementation has produced groundbreaking practical improvements - especially in robotic locomotion. In a similar fashion, we believe that focusing on a small-size yet meaningful system can help in isolating open control challenges in soft robotics, provide a test bench for experimenting with nonlinear control, and serve as a benchmark for different control approaches.

\section{A. Summary of contributions}

This work contributes to the state of the art in soft robotic control with: (i) an analytical model of soft robots with affine curvature, (ii) a low dimensional template model for under-actuated soft robots, (iii) the study of the main properties of this system, (iv) the closed loop stabilization of its unstable straight configuration by means of collocated and non collocated feedback linearization.

\section{B. Unit system}

Units will not be explicitly specified in the rest of the paper. All physical units may be assumed to be expressed in the MKS system, and angles in radian.

\section{MODEL}

\section{A. A preliminary note on the method}

The results that follow build on the ground laid by our previous work [13]. There we port the assumed mode technique [16] from the flexible link world to the soft one, by expressing the curvature of the soft robot through a polynomial expansion. We then derive a partially numerical model, and an analytical one under the further assumption of
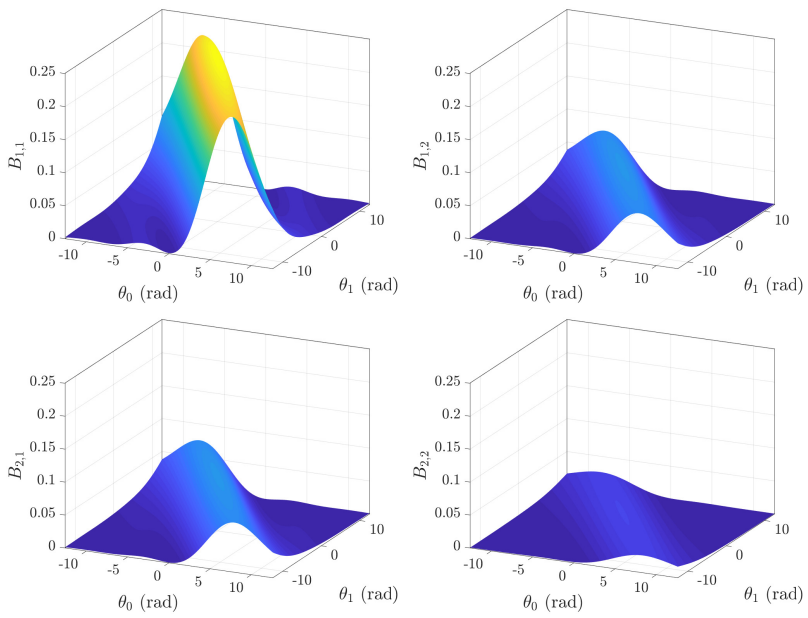

Fig. 3. Values of the components of the inertia matrix $B$, for an exhaustive range of configurations.
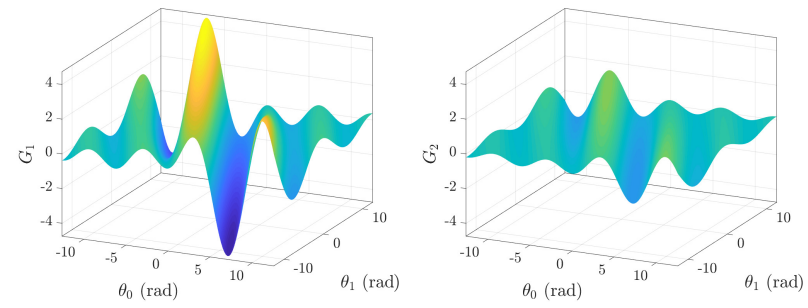

Fig. 4. Values of the gravity force field $G$ for an exhaustive range of configurations. Note that elastic forces are not included.

almost constant curvature. A similar modelling approach has been also proposed by [17]. There authors discuss the static case only and provide no control prospective. On the other hand, they come up with a much more general formulation in terms of admitted strains and actuation sources.

Despite reflecting a same philosophy, the results introduced by the following subsections substantially differ from [13]. Indeed, here we explore in depth what happens when the curvature function is affine rather than a generic polynomial, showing that under this assumption an exact model can be derived without further approximations.

\section{B. General definitions}

Consider a segment of soft material as in Fig. 1. Its length is $L$ and its thickness $D$. The structure is considered to be inextensible. Only planar motions are studied here. A coordinate $s \in[0,1]$ parametrizes the positions along the main axis of the pendulum, in such a way that $L s$ is the arc length of the path connecting the base to the point $s$ through the main axis. A reference frame $\left\{S_{s}\right\}$ is connected at each point $s$, therefore $\left\{S_{0}\right\}$ is the base frame and $\left\{S_{1}\right\}$ the tip frame. The relative orientation of all frames w.r.t. the base frame is zero when the system in straight. The posture of $\left\{S_{s}\right\}$ w.r.t. $\left\{S_{0}\right\}$ is $\left(x_{s}, y_{s}, \alpha_{s}\right) \in \mathbb{R}^{3}$, with $x_{s}$ and $y_{s}$ being the Cartesian coordinates of the frame's origin, and $\alpha_{s}$ the local orientation. A further coordinate $d \in\left[-\frac{1}{2},+\frac{1}{2}\right]$ is introduced to parametrize the remaining points within the segment. The Cartesian coordinates of a point $(s, d)$ in the global frame are called $\left(x_{s, d}, y_{s, d}\right)$, while in the local frame $\left\{S_{s}\right\}$ they are $(d, 0)$. This implies that the overall shape of the robot at each time $t$ is completely specified by the 


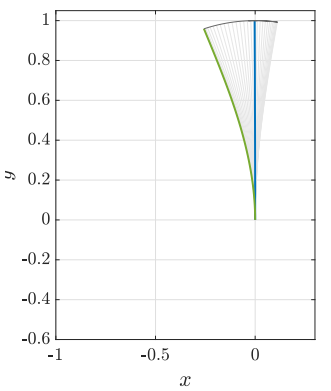

(a) Pendulum, $k=4$

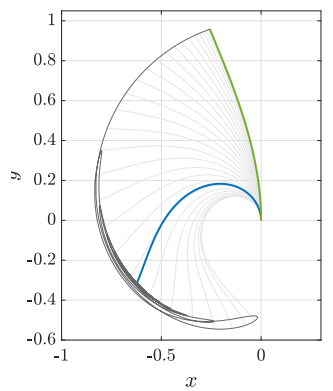

(c) Pendulum, $k=1$

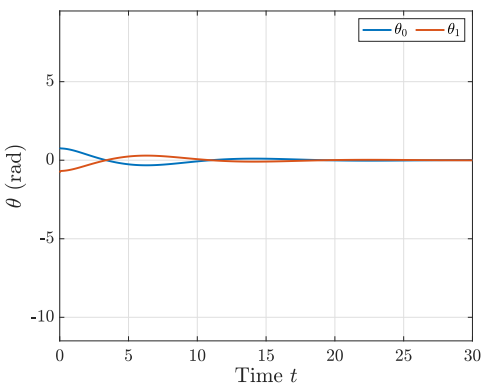

(b) Configuration, $k=4$

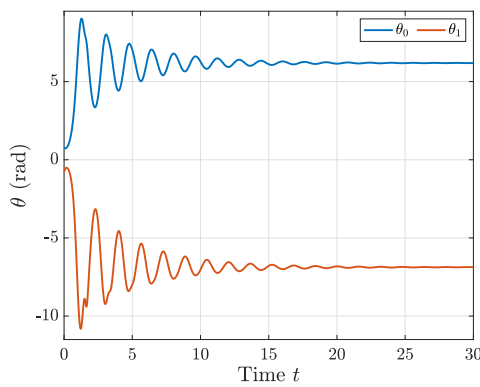

(d) Configuration, $k=1$
Fig. 5. Autonomous $(\tau \equiv 0)$ evolutions of the soft pendulum, for two different values of the stiffness $k$, and same initial condition $\theta(0)=$ $(\pi / 4,-\pi / 4)$. Panels (a) and (c) show the evolution of pendulum shape. The initial condition is shown in green, and the final in blue. Intermediate configurations are displayed in light gray. The evolution of the pendulum's tip is represented by a solid line of a darker grey. Panels (b) and (d) reports the time evolution of the configuration variables.

configuration of its central axis. This in turn is completely defined by the curvature at each point $\kappa_{s}(t):[0,1] \times \mathbb{R} \rightarrow \mathbb{R}$, see for example [18, Sec. 1.5].

From now on, we assume that $\kappa_{s}$ is well approximated by an affine function of the coordinate $s$

$$
\kappa_{s}(t)=\theta_{0}(t)+\theta_{1}(t) s,
$$

where $\theta_{0}$ and $\theta_{1}$ are the constant and linear components of $\kappa$ and they will serve as our configuration variables, with $\theta=\left(\theta_{0}, \theta_{1}\right)$ being the configuration vector. Therefore this model extends the constant curvature one [11], to which it is traced back for $\theta_{1} \equiv 0$.

\section{Kinematics}

We aim at expressing the posture of each material point as sole function of $\theta$. We start from $d=0$ (principal axis). The orientation is the integral of the curvature [18, Sec. 1.7]

$$
\alpha_{v}(t)=\int_{0}^{v} \kappa_{s}(t) \mathrm{d} s=\theta_{0}(t) v+\theta_{1}(t) \frac{v^{2}}{2},
$$

where $v$ is an auxiliary variable with the same meaning as $s$. The Cartesian coordinates can then be evaluated as

$$
x_{v}(t)=-L \int_{0}^{v} \sin \left(\alpha_{s}(t)\right) \mathrm{d} s, \quad y_{v}(t)=L \int_{0}^{v} \cos \left(\alpha_{s}(t)\right) \mathrm{d} s .
$$

These integrals can be solved by expanding the argument of sine and cosine, and integrating the terms separately. The result is as follows

$$
\begin{aligned}
\frac{x_{v}(t)}{L} & =\sin \left(\frac{\theta_{0}^{2}}{2 \theta_{1}}\right) \sqrt{\frac{\pi}{\theta_{1}}}\left[\mathrm{C}\left(\frac{\theta_{0}+v \theta_{1}}{\sqrt{\pi \theta_{1}}}\right)-\mathrm{C}\left(\frac{\theta_{0}}{\sqrt{\pi \theta_{1}}}\right)\right] \\
& -\cos \left(\frac{\theta_{0}^{2}}{2 \theta_{1}}\right) \sqrt{\frac{\pi}{\theta_{1}}}\left[\mathrm{~S}\left(\frac{\theta_{0}+v \theta_{1}}{\sqrt{\pi \theta_{1}}}\right)-\mathrm{S}\left(\frac{\theta_{0}}{\sqrt{\pi \theta_{1}}}\right)\right] .
\end{aligned}
$$

$$
\begin{aligned}
\frac{y_{v}(t)}{L}= & \cos \left(\frac{\theta_{0}^{2}}{2 \theta_{1}}\right) \sqrt{\frac{\pi}{\theta_{1}}}\left[\mathrm{C}\left(\frac{\theta_{0}+v \theta_{1}}{\sqrt{\pi \theta_{1}}}\right)-\mathrm{C}\left(\frac{\theta_{0}}{\sqrt{\pi \theta_{1}}}\right)\right] \\
& +\sin \left(\frac{\theta_{0}^{2}}{2 \theta_{1}}\right) \sqrt{\frac{\pi}{\theta_{1}}}\left[\mathrm{~S}\left(\frac{\theta_{0}+v \theta_{1}}{\sqrt{\pi \theta_{1}}}\right)-\mathrm{S}\left(\frac{\theta_{0}}{\sqrt{\pi \theta_{1}}}\right)\right],
\end{aligned}
$$

where we make use of the Fresnel integrals [19, Appendix A], i.e. $S(w)=\int_{0}^{w} \sin \left(\frac{\pi}{2} t^{2}\right) \mathrm{d} t, C(w)=\int_{0}^{w} \cos \left(\frac{\pi}{2} t^{2}\right) \mathrm{d} t$. It is worth underlying here that these functions are defined in the whole complex plane, and it can be proven by direct inspection that $S(i w)=-i S(w)$ and $C(i w)=i C(w)$. Therefore, $x_{v}$ and $y_{v}$ are always real numbers, despite $\theta_{1}$ appearing as square root. For what concerns the division by $\theta_{1}$, algebraic manipulations yield $\lim _{\theta_{1} \rightarrow 0} x_{v}(t)=$ $L\left(\cos \left(v \theta_{0}\right)-1\right) \backslash \theta_{0}$, and $\lim _{\theta_{1} \rightarrow 0} y_{v}(t)=L \sin \left(v \theta_{0}\right) \backslash \theta_{0}$. These are the expressions of Cartesian coordinates that we commonly find in the constant curvature literature [11]. These expressions are in turn well defined in the straight configuration of the segment $\lim _{\theta \rightarrow 0} x_{v}(t)=0$, $\lim _{\theta \rightarrow 0} y_{v}(t)=L v$. Fig. 2 reports a study of the segment's tip workspace, i.e. for $v=1$. Plots of the system in several configurations are also shown in panels (b) and (c), providing some examples of the versatility of the proposed kinematic description.

Finally, Cartesian coordinates of a generic point $(s, d)$ can be evaluated by directly applying the definition: $x_{v, d}=x_{v}+d D \cos \left(\theta_{0} v+\theta_{1} v^{2} / 2\right), y_{v, d}=y_{v}+$ $d D \sin \left(\theta_{0} v+\theta_{1} v^{2} / 2\right)$.

\section{Dynamics}

The inertia matrix is evaluated by integrating the contributions of all the infinitesimal mass elements

$$
B\left(\theta_{0}, \theta_{1}\right)=\int_{0}^{1} \int_{-\frac{1}{2}}^{+\frac{1}{2}} \rho(s, d) J_{s, d}^{\mathrm{T}} J_{s, d} \mathrm{~d} d \mathrm{~d} s,
$$

where $J_{s, d}=\nabla_{\theta}\left(x_{v, d}, y_{v, d}\right)$ is the Jacobian matrix mapping $\dot{\theta}$ to $\dot{x}_{v, d}$ and $\dot{y}_{v, d}$. Note that all elements of $J_{s, d}$ are function of both the states. Coriolis and centrifugal contributions $C(\theta, \dot{\theta}) \dot{\theta}$ can be evaluated from $B$ using standards procedures - e.g. Christoffel symbols.

The gravitational field is obtained as well by summing up all the contributions of the infinitesimal mass elements

$$
G(\theta)=\nabla_{\theta}^{\mathrm{T}} \int_{0}^{1} \int_{-\frac{1}{2}}^{\frac{1}{2}} \rho(s, d) g\left(\sin (\phi) x_{s, d}+\cos (\phi) y_{s, d}\right) \mathrm{d} d \mathrm{~d} s,
$$

where $\phi$ is a fixed value defining the direction of the field.

Finally, elastic, dissipative, and actuation forces are derived following the same steps as in [13], yielding to

$$
B(\theta) \ddot{\theta}+C(\theta, \dot{\theta}) \dot{\theta}+G(\theta)+k H \theta+\beta H \dot{\theta}=H\left[\begin{array}{l}
1 \\
0
\end{array}\right] \tau
$$

where $H \in \mathbb{R}^{2 \times 2}$ is the Hankel matrix $H_{i, j}=1 /(i+j-1)$, $k \in \mathbb{R}$ is the segment stiffness, and $d \in \mathbb{R}$ its damping.

\section{E. The soft inverted pendulum}

We call soft inverted pendulum with affine curvature a soft segment described by (8), and subject to a gravitational field aligned to the direction identified by the straight configuration of the Pendulum, oriented so to serve as a destabilizing force - in the inverse pendulum fashion. This is obtained for $\phi=0$, as shown in Fig. 1. In this case we 

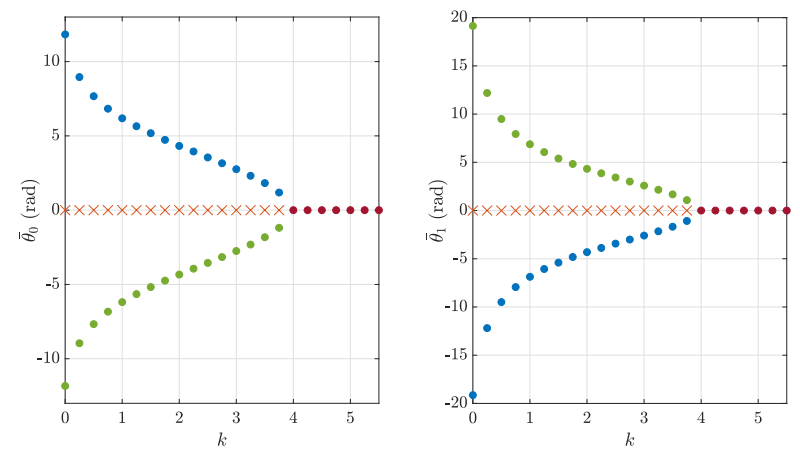

Fig. 6. Supercritical pitchfork bifurcation of equilibria to changes of stiffness $k$. Colors are used to identify the corresponding points in the two plots. Crosses identify unstable equilibria, while dots indicate stable ones.
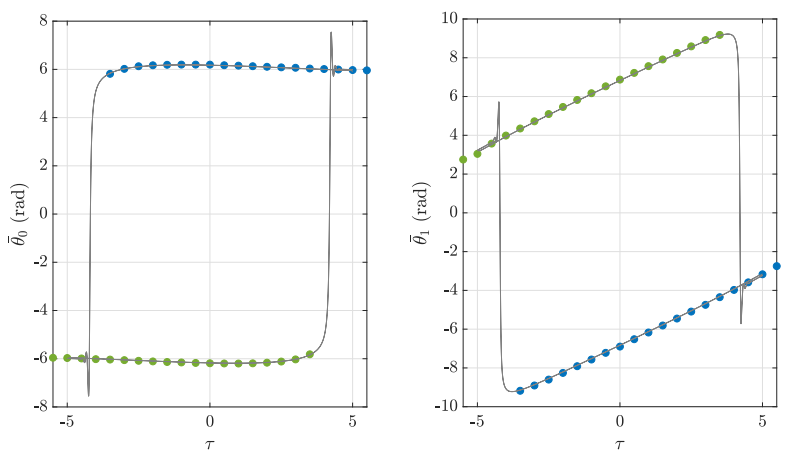

Fig. 7. Effect of a constant torque $\tau$ in shaping the equilibrium configurations of the system. For strong actions one of the two eqiulibria disappears. Colors are used to identify the corresponding points in the two plots. We report in light grey the cycle of hysteresis that we obtain when slowily varying the actuation from -5 to 5 .

have

$$
G(0,0)=(0,0)
$$

\section{F. Choice of inertia distribution}

The integrals (6) and (7) have closed form solution for several choices of the normalized ${ }^{1}$ density function $\rho(s, d)$, including the uniform distribution of mass $\rho \equiv m$. We consider here the soft pendulum mass to be concentrated at the tip of the segment, i.e. $\rho(s, d)=m \delta_{s-1}$ with $\delta_{w}$ being the Dirac's delta. Note that we cannot compress further our mass distribution to a point since the $y$ coordinate has null gradient around the straight configuration. As consequence the inertia matrix would loose rank. This is however solved including the rotational inertia introduced by the $d$ direction. Even within this simplifying assumption the explicit form of $B$ and $G$ are too long to be displayed (each element would occupy approximately a page). We resort instead to plotting their values in Figs. 3 and 4, for the choice of parameters introduced in the next example.

Example 1. Consider the soft pendulum described by (8) with $L=1, D=.1, m=1, g=9.81$, and $\beta=0.1$. The system starts from the initial condition $\theta(0)=(\pi / 4,-\pi / 4)$, $\dot{\theta}(0)=0$. Two values of $k$ are considered, i.e. medium stiffness 4 and low stiffness 1 . The resulting autonomous

\footnotetext{
${ }^{1}$ The unit of $\rho$ is the one of a weight, since $s$ and $d$ are normalized to be unit-less quantities. Note also that $\int_{0}^{1} \int_{-1 / 2}^{+1 / 2} \mathrm{~d} d \mathrm{~d} s=1$.
}
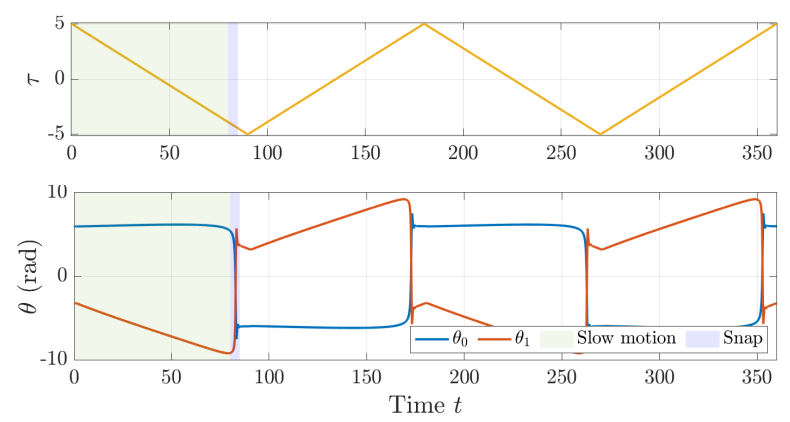

Fig. 8. Time progression of quasi static control action $\tau$ and its effect on $\theta$. The resulting evolution is a sequence of slow motions and sudden snaps spanning the whole workspace of the pendulum. The first two instances of this behaviour are highlighted with green and purple areas respectively.

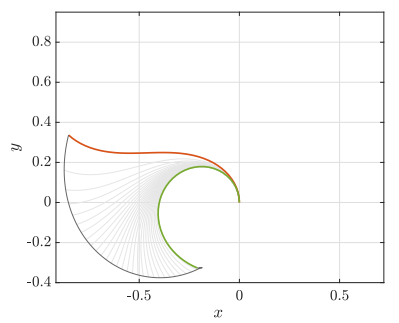

(a) Pendulum, $t \in(0,80)$

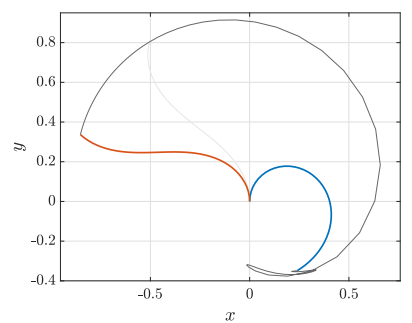

(b) Pendulum, $t \in(80,85)$
Fig. 9. As a response to an input slowly varying from -5 to 5 the pendulum first undergoes a slow deformation, shown in panel (a). The green line is the initial shape $(t=0)$, the red one to the final one $(t=80)$. Intermediate shapes are shown in light gray. When a critical value of $\tau$ is exceeded the pendulum presents catapult-like behaviour. It releases all the stored energy in a fast burst, and moves in few second to the opposite side of the workspace. This is shown by panel (b). The red line refers to the initial condition $(t=80)$, the blue one to the final one $(t=85)$.

$(\tau=0)$ evolution is obtained by integrating (8), and it is reported in Fig. 5.

\section{A STUDY OF EQUILIBRIA}

The soft inverted pendulum with affine curvature has a quite rich structure, that we briefly investigate in this section. Plugging the equilibrium conditions $(\dot{\theta}=0$ and $\ddot{\theta}=0)$ in (8) yields

$$
G_{1}(\bar{\theta})+\kappa \bar{\theta}_{0}+\frac{\kappa}{2} \bar{\theta}_{1}=\bar{\tau}, G_{2}(\bar{\theta})+\frac{\kappa}{2} \bar{\theta}_{0}+\frac{\kappa}{3} \bar{\theta}_{1}=\frac{\bar{\tau}}{2},
$$

where $\bar{\theta}$ is the equilibrium configuration and $\bar{\tau}$ the equilibrium torque.

\section{A. Origin}

Thanks to (9) we can immediately see that $(0,0)$ is always an equilibrium for $\bar{\tau}=0$, and for all possible values of $k, m, L, D$. Looking at the results of Example 1, we can expect the stability of this equilibrium to be dependent on how much the pendulum is soft. This intuition can be formalized by looking at the stiffness matrix

$$
K=\nabla_{\theta}(G(\theta)+k H \theta)_{\theta=(0,0)}=\left[\begin{array}{ll}
k-\frac{L g m}{3} & \frac{k}{2}-\frac{L g m}{8} \\
\frac{k}{2}-\frac{L g m}{8} & \frac{k}{3}-\frac{L g m}{20}
\end{array}\right] .
$$

According to Sylvester's criterion, $K$ is positive defined if and only if $K_{1,1}=k-\frac{L g m}{3}>0$, and $\operatorname{det}(K)=k^{2}-$ $\frac{13 \mathrm{Lgm}}{30} k+\frac{(\mathrm{Lgm})^{2}}{80}>0$. The first condition can be rewritten as $k /(\operatorname{Lgm})>1 / 3$, while the second translates into either $k /(\operatorname{Lgm})<(13-2 \sqrt{31}) / 60$, or

$$
k>\frac{13+2 \sqrt{31}}{60} \operatorname{Lgm} \text {. }
$$


Of all these conditions only the latter survives, being the more stringent. As expected, (11) tells us that the straight equilibrium is stable if the robot's stiffness is not too low when compared to the mass and the length.

\section{B. Other equilibria}

Example 1 also tells us that even when the origin becomes unstable the system still finds at least another stable equilibrium to converge to - as expected from a mechanical system subject to energy dissipation. Unfortunately, the complex nature of $G$ prevents the closed form solution of (10) for $\theta \neq(0,0)$. We will therefore fix the physical parameters of the system as in Example 1, and perform a bifurcation analysis of the equilibria when varying either $k$ or $\bar{\tau}$. Albeit we do not make any claim of generality here, it is worth mentioning that the same analysis was repeated for several choices of the physical parameters, with consistent results.

First, we look at the solutions of (10) when $\bar{\tau}=0$ and $k$ varies. Results are shown in Fig. 6. A supercritical pitchfork bifurcation [20, Sec. 2.3] can be observed in correspondence to the threshold defined by (11). If the pendulum is stiff enough only one equilibrium exists, corresponding to the straight configuration. When the pendulum gets too soft this equilibrium becomes unstable, and trifurcates originating two others symmetric and stable equilibria. This behavior persists even when $k$ reaches 0 .

We now look at the equilibria when a constant stiffness not verifying $(11)$ is selected $(k=1)$. We instead vary $\bar{\tau}$. This is shown in Fig. 7. As expected the two stable equilibria move while conserving their attractiveness when the input torque is modified. Interestingly, $\bar{\theta}_{0}$ stays almost constant, while $\bar{\theta}_{1}$ varies linearly. This at least until a critical value of $|\bar{\tau}|$ is reached, beyond which a blue-sky catastrophe manifests [20, Sec. 2.3], i.e. one of the two equilibria disappears. This behavior can give birth to an hysteresis cycle, by varying the input $\tau$ in an ample and slow enough fashion. Fig. 7 shows such cycle with a solid gray line.

Example 2. Consider the soft pendulum of Example 1, with $k=1$, and forced with a triangle wave with period 180 seconds, and amplitude 5. The system starts from the initial condition $\theta(0)=(2 \pi,-6 \pi), \dot{\theta}(0)=0$. Its evolution is shown in Fig. 8. The pendulum follows the motion of the closest equilbrium - storing energy in potential form. When the equilibrium disappears, the system is abruptly attracted to the only remaining equilibrium. The outcome is a sudden and fast release of the stored energy, resulting in the catapult-like trajectory shown by Fig. 9.

Behaviors as the one in Example 2 have been the object of study of continuum mechanics literature [21], as a product of infinite dimensional descriptions of the elastic rods. The fact that a so low dimensional model can produce such a complex behavior is somehow unexpected, and it is an aspect worth investigating in future work.

\section{FEEDBACK STABILIZATION}

We study here the stabilization of the straight configuration of the soft inverted pendulum, by means of partial feedback linearization.
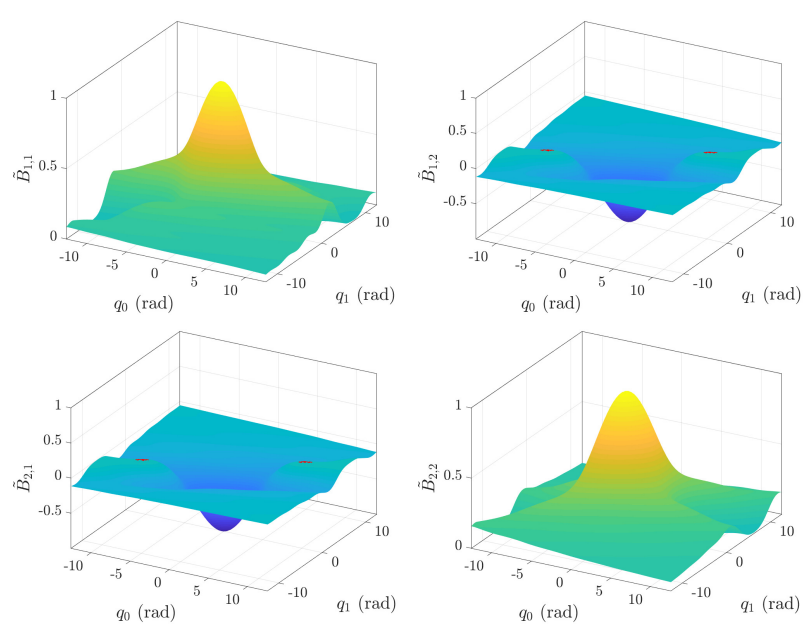

Fig. 10. Values of the components of the inertia matrix $\tilde{B}$, for an exhaustive range of configurations. The small areas in which the inertia coupling is lost (i.e. $B_{1,2}=0$ ) are highlighted in red in the picture.
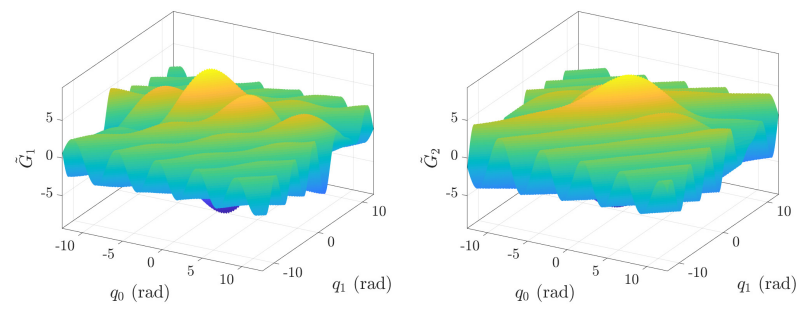

Fig. 11. Values of the gravity force field $\tilde{G}$, for an exhaustive range of configurations. Note that elastic forces are not included.

\section{A. Change of coordinates}

We want to clearly separate the system in an actuated portion and a completely under-actuated one, in the fashion of [14]. To this end, we introduce the following change of coordinates

$$
q_{0}=\theta_{0}+\frac{1}{2} \theta_{1}=\alpha_{1}, q_{1}=\frac{1}{2} \theta_{0}+\frac{1}{3} \theta_{1}=\int_{0}^{1} \alpha_{s} \mathrm{~d} s,
$$

where $q=\left(q_{0}, q_{1}\right)$ serves as the new configuration of the pendulum. By means of (12), Eq. (8) is modified into (note that $H$ is symmetric and positive defined)

$$
\tilde{B}(q) \ddot{q}+\tilde{C}(q, \dot{q}) \dot{q}+\tilde{G}(q)+\tilde{K} q+\tilde{D} \dot{q}=\left[\begin{array}{l}
\tau \\
0
\end{array}\right],
$$

where the new inertia matrix is $\tilde{B}(q)=H^{-1} B\left(H^{-1} q\right) H^{-1}$, and the new matrix collecting Coriolis and centrifugal effects is $\tilde{C}(q, \dot{q})=H^{-1} C\left(H^{-1} q, H^{-1} \dot{q}\right) H^{-1}$. Gravitational forces in the new coordinates are $\tilde{G}(q)=H^{-1} G\left(H^{-1} q\right)$. The new stiffness matrix is $\tilde{K}=k H^{-1} \succ 0$, and the new damping is $\tilde{D}=\beta H^{-1} \succsim 0$. We report in Figs. 10 and 11 the plots of $\tilde{B}$ and $\tilde{G}$, for the choice of parameters of Example 1. With the goal of writing more compact equations in the following, we define $h(q, \dot{q})=\tilde{C}(q, \dot{q}) \dot{q}+$ $\tilde{G}(q)+\tilde{K} q+\tilde{D} \dot{q}$. For the same reason, we will also omit dependencies on $q$ and $\dot{q}$ when possible.

\section{B. Collocated feedback}

We attempt here to stabilize the straight configuration by feedback linearizing the output $q_{0}=\alpha_{1}$. The first step towards this goal is to write the dynamics of $q_{0}$. This can 


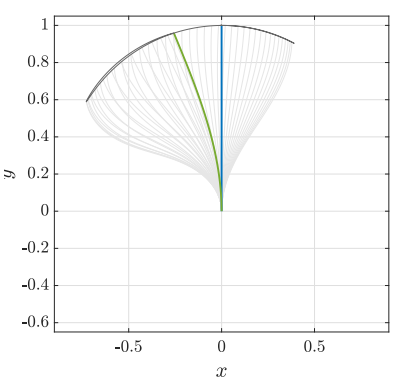

(a) Pendulum, $k=1$

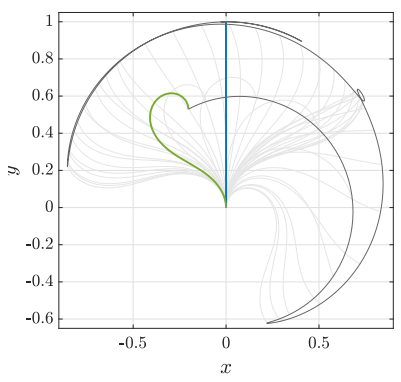

(e) Pendulum, $k=1$, far

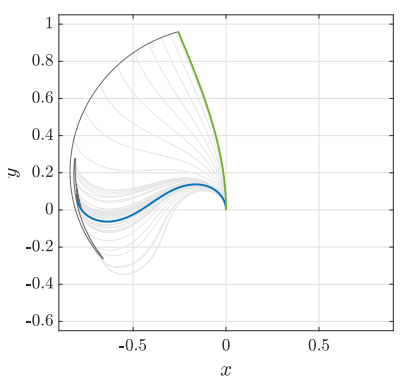

(i) Pendulum, $k=0.5$

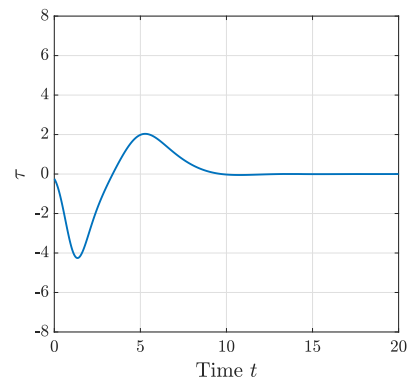

(b) Control action, $k=1$

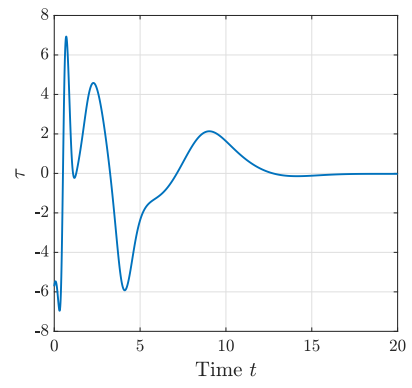

(f) Control action, $k=1$, far

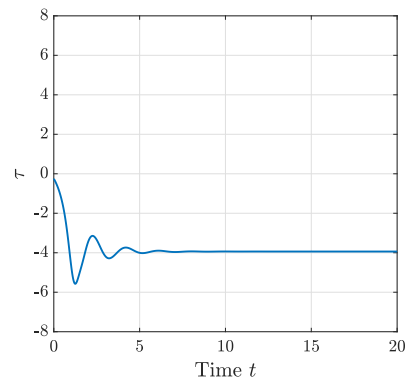

(j) Control action, $k=0.5$

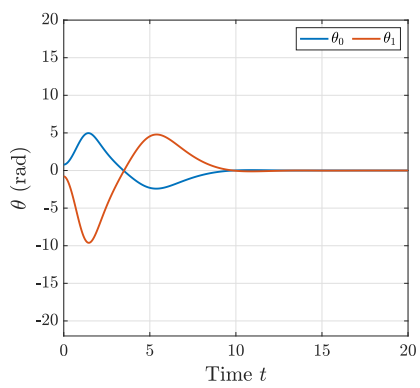

(c) Natural config., $k=1$

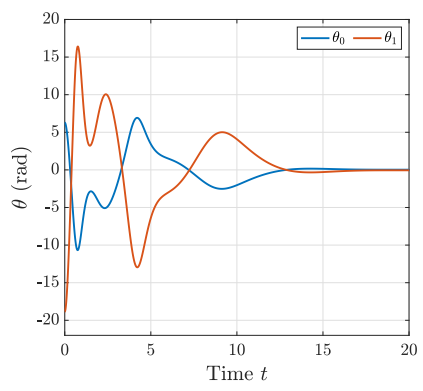

(g) Natural config., $k=1$, far

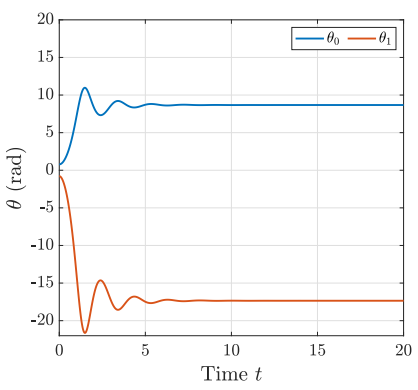

(k) Natural config., $k=0.5$

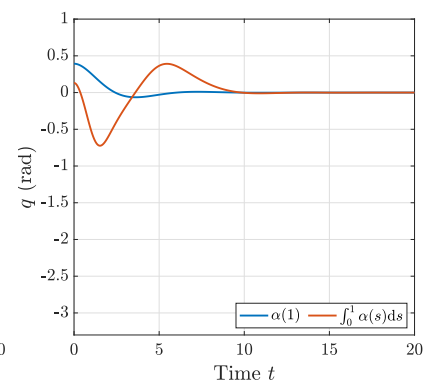

(d) Control config., $k=1$

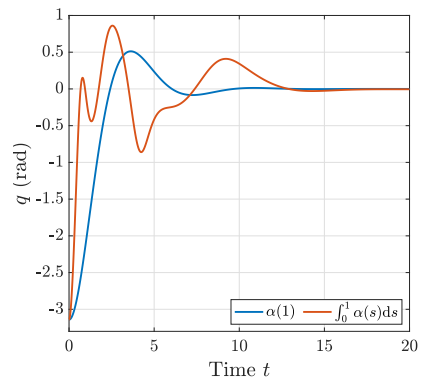

(h) Control config., $k=1$, far

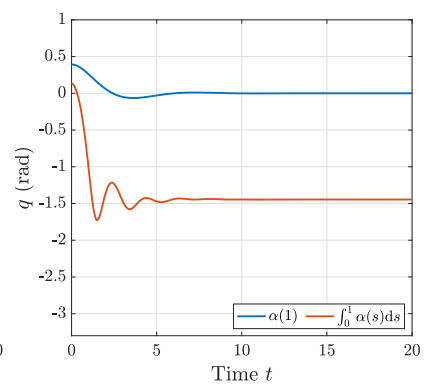

(1) Control config., $k=0.5$

Fig. 12. Results when closing the loop with collocated controller (15), combined with a PD regulator with unitary gains. Panels (a,e,i) display how the shape of the pendulum evolves. The initial condition is shown in green, and the final in blue. Intermediate configurations are displayed using light gray. The evolution of the pendulum's tip is represented by a solid line of a darker grey. Panels (b,f,j) report the control action, panels (c,g,k) the evolution in coordinates $\theta$, and $(\mathrm{d}, \mathrm{h}, \mathrm{l})$ in $q$. Note that the axes limits is coherent across simulations. Panels (a-d) depict the results for a value of stiffness $(k=1)$ verifying condition for asymptotic stability (18). The initial condition is $\theta(0)=(\pi / 4,-\pi / 4)$. Panels (e-h) present the results of the same closed loop, when initialized much further from the equilibrium, in $\theta(0)=(2 \pi,-3 \pi)$. Panels (i-1) report a simulation with stiffness under the critical threshold required for stability $(k=.5)$. The initial condition is again $\theta(0)=(\pi / 4,-\pi / 4)$.

be done by extracting $\ddot{q}_{1}$ from the second equation of (13), and substituting it in the first. The following second order differential equation results

$$
\left(\tilde{B}_{1,1}-\tilde{B}_{2,1}^{2} / \tilde{B}_{2,2}\right) \ddot{q}_{0}+\left(h_{1}-\left(\tilde{B}_{2,1} / \tilde{B}_{2,2}\right) h_{2}\right)=\tau \text {. }
$$

This equation can be feedback linearized by

$$
\tau=\left(h_{1}-\left(\tilde{B}_{2,1} / \tilde{B}_{2,2}\right) h_{2}\right)+\left(\tilde{B}_{1,1}-\tilde{B}_{2,1}^{2} / \tilde{B}_{2,2}\right) u
$$

where $u$ is an extra control action to be defined later. Note that this feedback loop is always well defined since $\tilde{B}_{2,2} \neq$ 0 , being a principal minor of an inertia matrix. The resulting closed loop dynamics is

$$
\ddot{q}_{0}=u, \quad \tilde{B}_{2,2} \ddot{q}_{1}+h_{1}=-\tilde{B}_{1,2} u .
$$

According to [14, Theorem 1], the stability of this dynamics can be analyzed by looking separately at the convergence of $q_{0}$, and at the dynamics of $q_{1}$ under the assumption that $q_{0} \equiv 0$, i.e. the zero-dynamics.

To stabilize $q_{0}$ we consider a simple PD regulator acting on the linearized dynamics only, $u=-\varphi_{\mathrm{P}} q_{0}-\varphi_{\mathrm{D}} \dot{q}_{0}$, where $\varphi_{\mathrm{P}}$ and $\varphi_{\mathrm{D}}$ are positive scalar gains. This control action brings $q_{0}$ to 0 exponentially fast, starting from any arbitrary initial condition.

Since $u(0,0)=0$, the zero-dynamics is obtained by setting to 0 all the instances of $q_{0}$ and $\dot{q}_{0}$ in the second equation of (13). The result is $\tilde{B}_{2,2}\left(0, q_{1}\right) \ddot{q}_{1}+\tilde{C}_{2,2}\left(0, q_{1}, 0, \dot{q}_{1}\right) \dot{q}_{1}+$ $\tilde{G}_{2}\left(0, q_{1}\right)+\tilde{K}_{2,2} q_{1}+\tilde{D}_{2,2} \dot{q}_{1}=0$. The stability of this system can be proven by using $V\left(q_{1}, \dot{q}_{1}\right)=\tilde{B}_{2,2}\left(0, q_{1}\right) \dot{q}_{1}^{2} / 2+$ $\int_{0}^{q_{1}} \tilde{G}_{2}\left(0, q^{*}\right) \mathrm{d} q^{*}+\tilde{K}_{2,2} q_{1}^{2} / 2$ as Lyapunov candidate. Its derivative is $\dot{V}=-\tilde{D}_{2,2} \dot{q}_{1}^{2} \leq 0$, and the thesis follows by invoking LaSalle's invariance principle. Note however that $V\left(q_{1}, \dot{q}_{1}\right)$ needs to be positive defined. This is not trivially provable for generic choice of the system parameters, due to the complex form of $\tilde{G}$. So, we settle for proving the positivenes locally by looking at the stiffness

$$
\frac{\partial}{\partial q_{1}}\left(\tilde{G}_{2}\left(0, q_{1}\right)+\tilde{K}_{2,2} q_{1}\right)_{q_{1}=0}=12 k-\frac{12}{10} m g L>0 .
$$




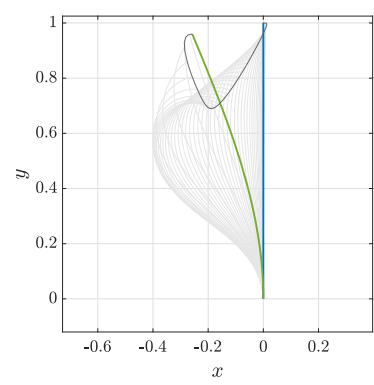

(a) Pendulum, $k=0.25$

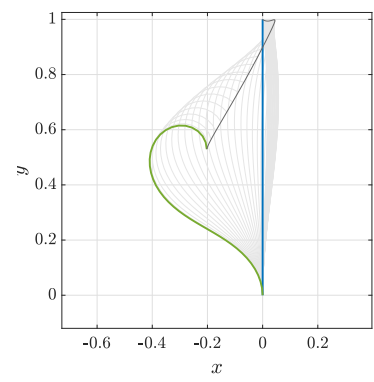

(e) Pendulum, $k=0.25$, far

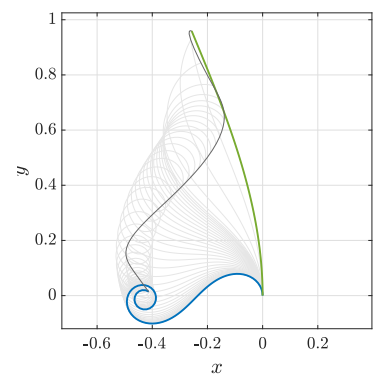

(i) Pendulum, $k=0.1$

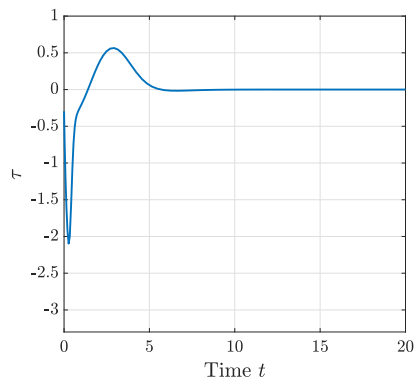

(b) Control action, $k=0.25$

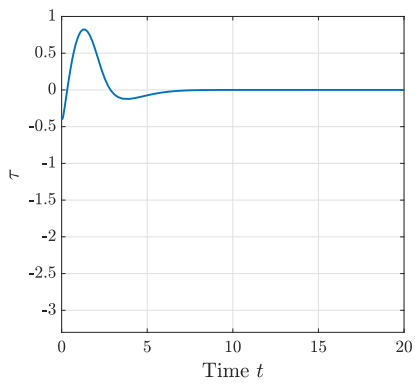

(f) Control action, $k=0.25$, far

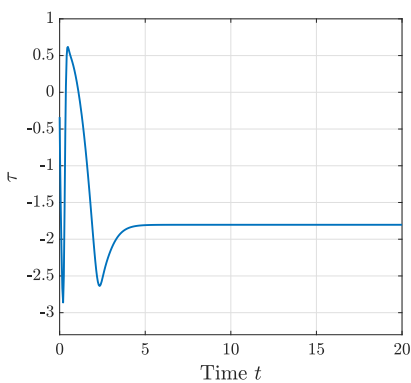

(j) Control action, $k=0.1$

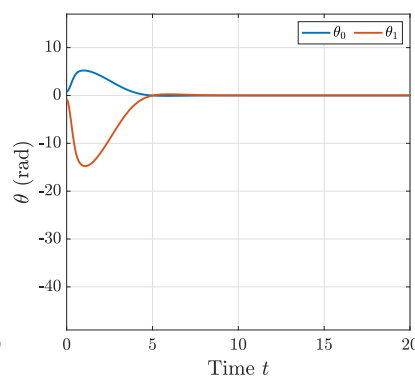

(c) Natural config., $k=0.25$

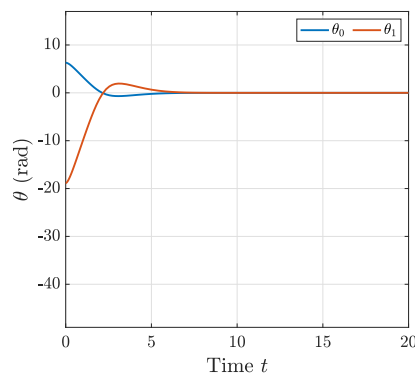

(g) Natural config., $k=0.25$, far

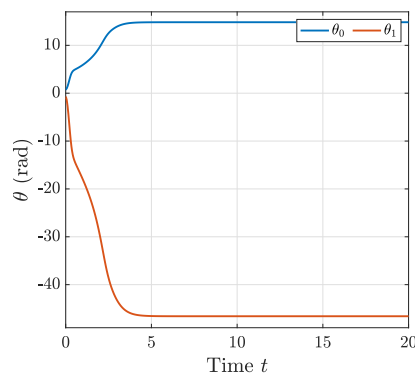

(k) Natural config., $k=0.1$

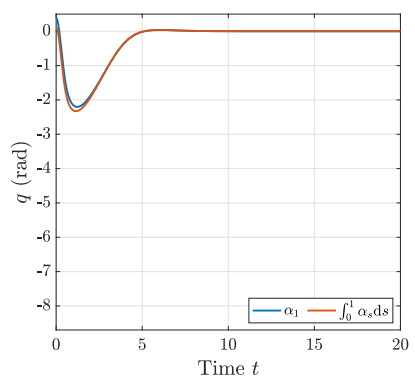

(d) Control config., $k=0.25$

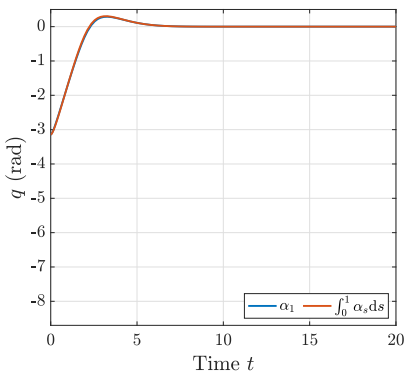

(h) Control config., $k=0.25$, far

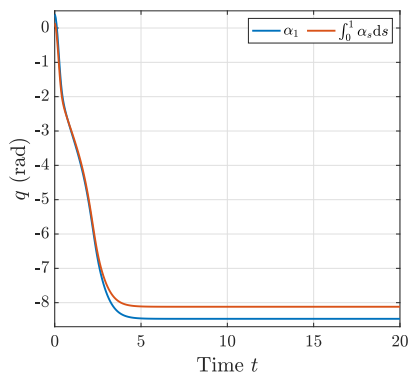

(1) Control config., $k=0.1$

Fig. 13. Effect of the non collocated control loop (20), with $u$ selected as an LQR designed on the linearized system. Panels (a,e,i) depict how the shape of the pendulum evolves. The initial condition is in green, the final in blue. Intermediate configurations are in light gray. The progression of the pendulum's tip is marked with a solid line of a darker grey. Panels $(\mathrm{b}, \mathrm{f}, \mathrm{j})$ depict the control action, panels $(\mathrm{c}, \mathrm{g}, \mathrm{k})$ the evolution in coordinates $\theta$, and $(\mathrm{d}, \mathrm{h}, \mathrm{l})$ in coordinates $q$. Note that the axes limits is coherent across simulations. Panels (e-h) show the behavior of the closed loop when the stiffness $(k=0.25)$ is substantially lower than the minimum admitted by the collocated controller. The initial condition is the usual $\theta(0)=(\pi / 4,-\pi / 4)$. Panels (i-k) are the same but with $\theta(0)=(2 \pi,-3 \pi)$. Panels $(\mathrm{m}-\mathrm{p})$ report a simulation with a very low stiffness $(k=0.1)$, for which even the non collocated control loop fails (even if locally the equilibrium is still attractive). The initial condition is again $\theta(0)=(\pi / 4,-\pi / 4)$.

At the same time we want the system in open-loop to be unstable, otherwise there is no point in using a stabilizing controller. Combining (11) and (17) yields

$$
\frac{13+2 \sqrt{31}}{60}>\frac{k}{g m L}>\frac{1}{10} \text {. }
$$

This equation defines the interval within which a collocated action can be both useful and effective. If the system is too soft, there is no chance of stabilizing the equilibrium in this way, and we must resort to something even more sophisticated $^{2}$.

Example 3. Consider the soft pendulum of Example 1, with $k=1$ or $k=0.5$, and controlled by (15) with $\varphi_{\mathrm{P}}=1$ and $\varphi_{\mathrm{D}}=1$. We consider the two usual starting configurations $\theta(0)=(\pi / 4,-\pi / 4)$ and $\theta(0)=(2 \pi,-6 \pi)$, both with $\dot{\theta}(0)=0$. The result is reported in Fig. 12 .

${ }^{2}$ For the sake of space, we do not consider a full state feedback for $u$.

\section{Non collocated feedback}

We consider here the possibility of feedback linearizing the output $q_{1}=\int_{0}^{1} \alpha_{s} \mathrm{~d} s$. The strategy is non collocated since the control action acts on $q_{0}$, and the controller has to influence $q_{1}$ through the dynamics [14].

We start by making explicit $\ddot{q}_{0}$ from the first equation of (13), and we substitute the result of this operation in the second equation. This procedure results in

$$
\left(\tilde{B}_{1,2}^{2}-\tilde{B}_{1,1} \tilde{B}_{2,2}\right) \ddot{q}_{1}+\left(\tilde{B}_{1,2} h_{1}-\tilde{B}_{1,1} h_{2}\right)=\tilde{B}_{1,2} \tau,
$$

from which the feedback linearizing loop is trivially derived as

$$
\tau=\left(h_{1}-\left(\tilde{B}_{1,1} h_{2}\right) / \tilde{B}_{1,2}\right)+\left(\tilde{B}_{2,1}-\left(\tilde{B}_{1,1} \tilde{B}_{2,2}\right) / \tilde{B}_{1,2}\right) u .
$$

Note that this control action makes sense only if $\tilde{B}_{1,2} \neq 0$ (so-called inertial coupling). This condition holds true for almost all the configurations of the soft pendulum, with the exception of the small areas shown in red in Fig. 10. 
Combining (13) and (20) yields

$$
\tilde{B}_{2,1} \ddot{q}_{0}=-h_{2}-\tilde{B}_{2,2} u, \quad \ddot{q}_{1}=u .
$$

This time a simple PD control on $q_{1}$ is not sufficient since the zero dynamics for $q_{1} \equiv 0$ and $u \equiv 0$ is $\ddot{q}_{0}=0$, which is polynomially unstable. Hence, we proceed to a local analysis of the complete dynamics (21) by linearizing the equations around the equilibrium $(0,0)$. The resulting linear system is sufficiently compact to be presented explicitly

$$
\dot{\eta}=\left[\begin{array}{cccc}
0 & 0 & 1 & 0 \\
0 & 0 & 0 & 1 \\
\frac{-6 k+\frac{11}{10} L g m}{L^{2} m} & \frac{12 k-\frac{12}{10} L g m}{L^{2} m} & -\frac{6 \beta}{L^{2} m} & \frac{12 \beta}{L^{2} m} \\
0 & 0 & 0 & 0
\end{array}\right] \eta+\left[\begin{array}{l}
0 \\
0 \\
1 \\
1
\end{array}\right] u,
$$

where $\eta \in \mathbb{R}^{4}$ describes small perturbations of $(q, \dot{q})$ around $(0,0)$. The system is always unstable, with two poles in 0 and the other two in $\left( \pm \sqrt{\frac{22 g L^{3} m^{2}}{5}-24 k L^{2} m+36 \beta^{2}}-\right.$ $6 \beta) /\left(2 L^{2} m\right)$. To see if the equilibrium can be locally stabilized we calculate the reachability matrix

$$
\left[\begin{array}{cccc}
0 & -1 & \frac{18 \beta}{L^{2} m} & r_{1} \\
0 & 1 & 0 & 0 \\
-1 & \frac{18 \beta}{L^{2} m} & r_{1} & r_{2} \\
1 & 0 & 0 & 0,
\end{array}\right]
$$

with $r_{1}=-\left(0.1 g L^{3} m^{2}-6 k L^{2} m+36 \beta^{2}\right) /\left(L^{4} m^{2}\right)$, and $r_{2}=36 \beta\left(g L^{3} m^{2}-10 k L^{2} m+30 \beta^{2}\right) /\left(5 L^{6} m^{3}\right)$. This matrix is full rank for all the values of the physical parameters verifying ${ }^{3} L^{3} g^{2} m^{2}-120 L^{2} g k m+3600 L k^{2}-$ $3600 \beta^{2} g \neq 0$. Therefore almost all the choices of physical parameters admit a complete pole placement of all the poles of $A$, leading to a locally asymptotically stable closed loop behavior.

Example 4. Consider the soft pendulum of Example 1, with $k=0.25$ or $k=0.1$, and controlled through (20) and $u=$ $\gamma_{P} q+\gamma_{D} \dot{q}$. The gains $\gamma_{P}$ and $\gamma_{D}$ are such to minimize the $L Q$ index $\int_{0}^{\infty} q^{\mathrm{T}} q+\dot{q}^{\mathrm{T}} \dot{q}+u^{\mathrm{T}} u \mathrm{~d} t$. We consider the two usual starting configurations $\theta(0)=(\pi / 4,-\pi / 4)$ and $\theta(0)=$ $(2 \pi,-6 \pi)$, both with $\dot{\theta}(0)=0$. The result is reported in Fig. 13.

\section{CONCLUSiOnS}

This paper introduced an underactuated low dimensional mechanical model - the inverted elastic pendulum - designed to serve as a template for soft robot control. This system proved to have a rich nonlinear behavior (bifurcations, hystereses), and presents unique challenges from the control standpoint. Nonlinear feedback regulators have been proposed based on partial feedback linearization. Despite not being always implementable in the practice, results obtained with this technique allow to establish upper bounds to the performance that controller relying less on the perfect knowledge of the model can hope to achieve. Interestingly, the kind of control approaches that can be implemented depends on the degree of softness of the system. Future work will include (i) investigating simpler control techniques, (ii) attacking the trajectory tracking problem, (iii) extending these results to full fledged soft robots.

\footnotetext{
${ }^{3}$ This condition is derived through straightforward manipulations of the matrix's determinant.
}

\section{REFERENCES}

[1] C. Della Santina, M. G. Catalano, and A. Bicchi, Soft Robots. Berlin, Heidelberg: Springer Berlin Heidelberg, 2020 (in press).

[2] D. Huang and J.-X. Xu, "Steady-state iterative learning control for a class of nonlinear pde processes," Journal of Process Control, vol. 21, no. 8, pp. 1155-1163, 2011.

[3] A. Mironchenko, I. Karafyllis, and M. Krstic, "Monotonicity methods for input-to-state stability of nonlinear parabolic pdes with boundary disturbances," SIAM Journal on Control and Optimization, vol. 57, no. 1 , pp. 510-532, 2019.

[4] D. C. Rucker and R. J. Webster III, "Statics and dynamics of continuum robots with general tendon routing and external loading," IEEE Transactions on Robotics, vol. 27, no. 6, pp. 1033-1044, 2011.

[5] T. G. Thuruthel, E. Falotico, M. Manti, and C. Laschi, "Stable open loop control of soft robotic manipulators," IEEE Robotics and Automation Letters, vol. 3, no. 2, pp. 1292-1298, 2018.

[6] H. Wang, B. Yang, Y. Liu, W. Chen, X. Liang, and R. Pfeifer, "Visual servoing of soft robot manipulator in constrained environments with an adaptive controller," IEEE/ASME Transactions on Mechatronics, vol. 22 , no. 1 , pp. 41-50, 2016.

[7] S. Grazioso, G. Di Gironimo, and B. Siciliano, "A geometrically exact model for soft continuum robots: The finite element deformation space formulation," Soft robotics, 2018.

[8] H. Sadati, S. E. Naghibi, A. Shiva, S. Zschaler, D. Rucker, M. Brendan, L. Renson, K. Althoefer, T. Nanayakkara, H. Hauser, and I. D. Walker, "Tmtdyn: A matlab package for modeling and control of hybrid rigid-continuum robots based on discretized lumped system and reduced order models," The International Journal of Robotics Research, 2019.

[9] B. Deutschmann, A. Dietrich, and C. Ott, "Position control of an underactuated continuum mechanism using a reduced nonlinear model," in 2017 IEEE 56th Annual Conference on Decision and Control (CDC), pp. 5223-5230, IEEE, 2017.

[10] V. Falkenhahn, A. Hildebrandt, R. Neumann, and O. Sawodny, "Model-based feedforward position control of constant curvature continuum robots using feedback linearization," in Robotics and Automation (ICRA), 2015 IEEE International Conference on, pp. 762767, IEEE, 2015

[11] C. Della Santina, R. K. Katzschmann, A. Bicchi, and D. Rus, "Modelbased dynamic feedback control of a planar soft robot: trajectory tracking and interaction with the environment," The International Journal of Robotics Research, p. 0278364919897292, 2019.

[12] M. Thieffry, A. Kruszewski, T.-M. Guerra, and C. Duriez, "Trajectory tracking control design for large-scale linear dynamical systems with applications to soft robotics," IEEE Transactions on Control Systems Technology, 2019.

[13] C. Della Santina and D. Rus, "Control oriented modeling of soft robots: the polynomial curvature case," IEEE Robotics and Automation Letters, vol. 5, no. 2, pp. 290-298, 2019.

[14] M. W. Spong, "Partial feedback linearization of underactuated mechanical systems," in Proceedings of IEEE/RSJ International Conference on Intelligent Robots and Systems (IROS'94), vol. 1, pp. 314321, IEEE, 1994.

[15] I. Fantoni and R. Lozano, Non-linear control for underactuated mechanical systems. Springer Science \& Business Media, 2002.

[16] A. De Luca and B. Siciliano, "Inversion-based nonlinear control of robot arms with flexible links," Journal of guidance, control, and dynamics, vol. 16, no. 6, pp. 1169-1176, 1993.

[17] F. Renda, C. Armanini, V. Lebastard, F. Candelier, and F. Boyer, "A geometric variable-strain approach for static modeling of soft manipulators with tendon and fluidic actuation," IEEE Robotics and Automation Letters, 2020.

[18] M. P. Do Carmo, Differential geometry of curves and surfaces: revised and updated second edition. Courier Dover Publications, 2016.

[19] L. Debnath and D. Bhatta, Integral transforms and their applications. CRC press, 2014.

[20] A. H. Nayfeh and B. Balachandran, Applied nonlinear dynamics: analytical, computational, and experimental methods. John Wiley \& Sons, 2008.

[21] C. Armanini, F. Dal Corso, D. Misseroni, and D. Bigoni, "From the elastica compass to the elastica catapult: an essay on the mechanics of soft robot arm," Proceedings of the Royal Society A: Mathematical, Physical and Engineering Sciences, vol. 473, no. 2198, p. 20160870, 2017. 